\title{
Modeling and Simulation of the Passive Regional Satellite Navigation System
}

\author{
Chen Zhonggui, Zhang Zhi, Liao Ying, Su Tianxiang \\ College of Aerospace and Material Engineering \\ National University of Defense Technology \\ Changsha 410073,China \\ zznavy83@yahoo.com.cn
}

\begin{abstract}
The passive regional satellite navigation system (PRSNS) becomes a significant topic for civil and military services due to the limit of outlays and geography factors. The research on the modeling and simulation (M\&S) of PRSNS is to study the key technology and operation principle. The paper discussed how to build up the simulation system of PRSNS. The system included satellite constellation, tracking station, data processing center (DPC), feeding station, users' application, information management, surveillance and visualization, etc. And the system is divided into two major logical parts and three physical layers to reduce the dependence among the components in order to quicken the programming process. As an example, a simulation system of PRSNS is constructed, which adopts a satellite constellation that is composed of geostationary earth orbit (GEO) satellites, inclined geosynchronous orbit (IGSO) satellites and median earth orbit (MEO) satellites and covers the Pacific-Asia, Indian Ocean and Middle East. The efficiency of the simulation system and the feasibility of PRSNS are verified by simulated static positioning and dynamic navigating.
\end{abstract}

Keywords-Satellite navigation system, Modeling and simulation (M\&S), Satellite constellation

\section{INTRODUCTION}

Unlike GPS, GLONASS and GALILEO[1], PRSNS covers local area and only can navigate for some regional area. But it is still composed of satellites constellation part in space, control part on the ground and users application[2]. Owning to complicated structure, high performance, longtime operation, high reliability and all-weather service, the system design of PRSNS is required strictly. So it needs simulation and evaluation system to carry out need analysis, top-level design, system construction, system maintenance in order to assign task, compare design, simulate fault, system upgrade [3].

\section{SYSTEM REALIZATION}

The simulation system of PRSNS is complicated and distributed that its structure must be carefully arranged to complete the task [4]. The simulation system of PRSNS is divided into two major logical parts and three physical layers.
The two logical parts are Simulation System and Simulation Application. The whole system is connected by fiber Ethernet, so that it can get the highest data transfer rate. The two parts division can help us focus our attention on the most important part, the simulation system of PRSNS, in the M\&S assignments.

All the above is about problem processing in the logic view, which can simplify the complication of the issue in our mind. And the whole simulation system is divided into three physical layers, which can quicken the programming process and reduce the dependence among the components. They are Numerical Value Computation Layer, Data Exchange Layer and Man-machine Conversation Layer.

- Numerical Value Computation Layer: this layer is the central part of the

simulation system of PRSNS. It is in charge of the numerical value computation of the all the mathematic models, such as Satellite Orbit Models, Planets' Gravitation Models and Time Computation Models, etc. The main task of this part is about algorithm's realization and improvement. It makes the designer and programmer focus on the validity and creditability of the models and programs.

- Data Exchange Layer: this layer is in charge of the data transfer form one

to another and the logic harmony between each processes or simulation programs. It is focused on how to use the tools HLA/RTI offered to improve data distribution, so that we easily isolate each model and reconstruct the simulation system. The main task of this part is to establish the Federation, which may include the FOM/SOM Development, Federation Management, Time Management, Declaration Management, Object Management, Ownership Management and Data Distribution Management, etc.

- Man-machine Conversation Layer: this layer is the interface between the

simulation system and users. Its main task is Data Display, Storage, Users' Command Response, Database Management, and Network State Monitor, etc. It is focused on how to facilitate the users to use and control the simulation system to fulfill their own task.

\section{SimULATION SYSTEM DESIGN}

\section{A. Satellites constellation}

As a passive satellite navigation system, the satellites in the space mainly broadcast the position and velocity of the satellites and navigation information, so that the user 
application can position and navigate, and track stations can observe. Some simulations as follows are carried out.

1). The satellites atomic clock simulation

An atomic clock on the satellites is simulated to output the satellites' atomic time, which needs to take account of the time shift and relativity effect in the space.

2). Precise orbit dynamical simulation of navigating satellites

Precise orbit dynamical simulation calculates the forces acting on the satellites and computes the satellites' orbit for satellite orbit dynamical simulation. Owning to the high orbit of the navigating satellites, the perturbation of atmospheric drag can be neglected, and the gravitational effects of the earth, Lunar and solar gravitational force perturbations, and solar radiation pressure are taken into account. So the satellites' attitude, mass, shape and area, solar panels area and attitude need to be input, and the satellites orbit is integrated by KSG integrator and RungeKutta integrator.

3). Satellites attitude simulation

It simulates the satellites orientation mode, set up satellites attitude kinematic model, and provide the satellites attitude.

\section{B. Tracking stations}

Track station's function tracks satellites to get satellite laser rang (SLR), P-mode pseudo-range, C\A-mode pseudorange, satellite clock error, and pseudo-range error information and to serve DPC to determine satellite orbit, compute the satellite ephemeris and clock error and NTM. Pseudo-range error computation is to inspect and validate the navigation system to improve the positioning RAIM and reliability.

1). The track station atomic clock simulation

An atomic clock on the track station is simulated to output the atomic time, which is taken account of the time shift.

2). The atomic clock contrast simulation between satellite and track station

The atomic clock contrast simulation is to get clock error between satellite and track station, which will be sent to DPC to calculate the satellite clock error.

3). SLR, P-mode pseudo-range and C $\backslash A$-mode pseudorange simulation

SLR, P-mode pseudo-range and C\A-mode pseudorange simulation between satellite and track station needs to compute the ionospheric refraction, the tropospheric refraction, station and satellite eccentricity effect, the earth rotation effect, multi-path effect, satellite clock error and receiver clock error effect. So the track stations' position in the earth core coordinate system, weather condition, station and satellite eccentricity parameter, double carrier wave frequencies and the laser frequency are input parameters.

4). Weather condition simulation in track stations

It is to simulate temperature, humid and air pressure in track station.

5). Pre-process of observations
Pre-process of observations includes error correction and data smooth. the ionospheric refraction correction v the tropospheric refraction correction 、 satellite and station eccentricity correction, the earth rotation effect is carried out for SLR, P-mode pseudo-range and C\A-mode pseudorange, the observations is smoothed by roust least square method.

\section{6). Satellite ephemeris demodulation}

It is to demodulate satellite ephemeris from NTM and then to calculate the satellite position, velocity, clock error and RAIM.

\section{System assessment}

In order to assess the performance of PRSNS, the following simulation is accomplished: Satellites constellation deployment and cover area; the single point positioning precision; the position and velocity precision for navigation; timing service precision, the relative positioning precision between the users application; Orientation precision; Satellite ephemeris and clock error precision assessment; Satellite attitude effect on navigation performance assessment; the navigation precision change assessment in space with time; choice assessment of the tracking stations; the satellite force perturbations model precision assessment; satellite constellation figure deformation assessment with time; and satellite constellation maintenance assessment.

\section{EXAMPLE AND ANALYSIS}

As an example, a simulation system of PRSNS is constructed, which adopts a satellite constellation that is composed of 5 geostationary earth orbit (GEO) satellites, 6 inclined geosynchronous orbit (IGSO) satellites and 4 median earth orbit (MEO) satellites. The longitude of 5 satellites distributes from 45 to 170 degree. There are 3 IGSO satellites that follow the same ground track like the number 8 which longitude of ascending node is 80degree and other 3 IGSO satellites on another same ground track which longitude is 140 degree. Therefore PRSNS covers the Pacific-Asia, Indian Ocean and Middle East and 12 tracking stations which are located in the same area are simulated. PRSNS constellation is presented in Figure 1, and tracking stations deployment and satellites ground track in Figure 2.

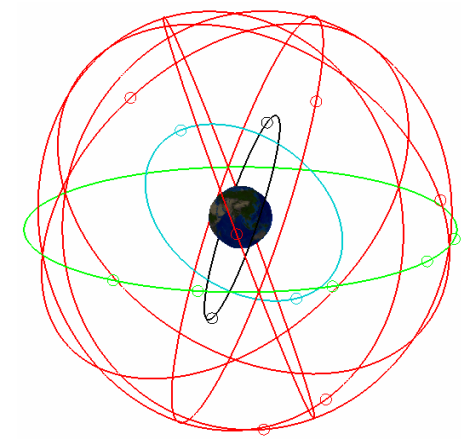

Figure 1. PRSNS constellation 


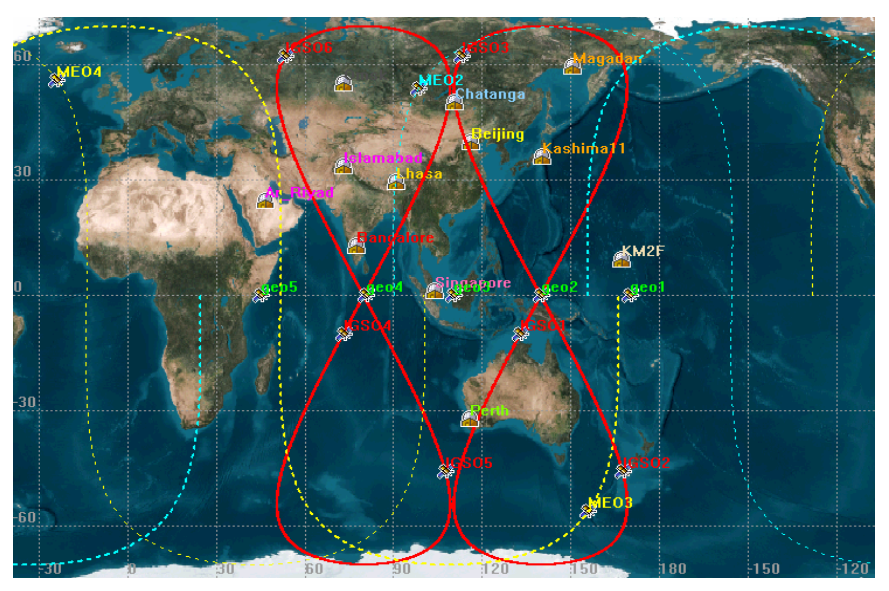

Figure 2. Tracking stations deployment and satellites ground track

The satellite orbit is determined by robust extended kalman filter with P-mode pseudo-ranges, and the orbit precision is shown in Figure 3 5.

The figures show that when kalman filter gets convergence, GEO orbit determination precision is about $2 \mathrm{~m}$, MEO 5m, and MEO 10m. But it spends a long time for MEO orbit determination to converge. This is because the MEO satellites can only be observed by tracking stations in part time, while the GEO satellites can be observed by tracking stations in whole arc.

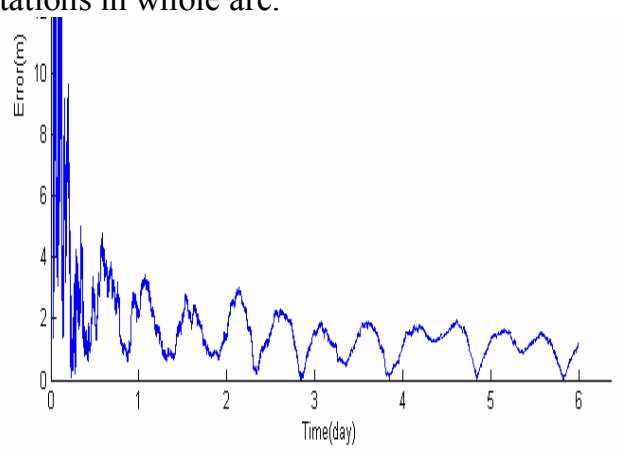

Figure 3. GEO orbit determination precision

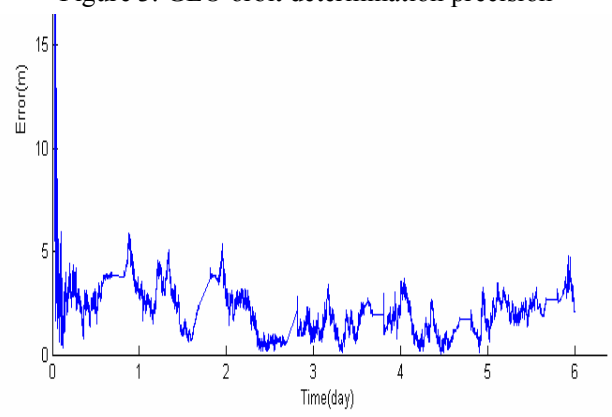

Figure 4. IGSO orbit determination precision

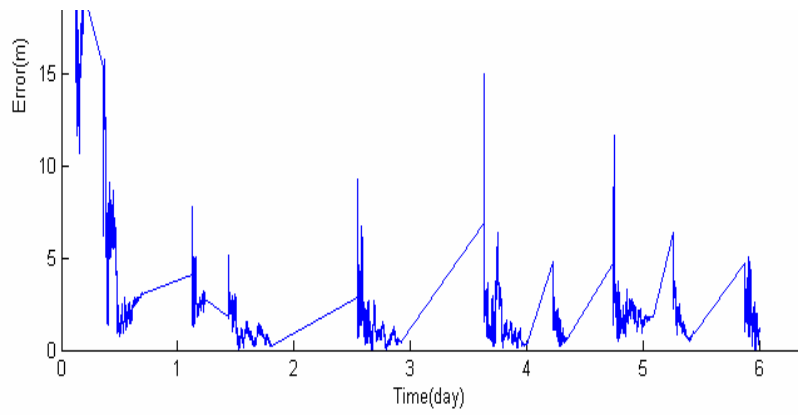

Figure 5. MEO orbit determination precision

When the positioning simulation is assessed, the PRSNS gets positioning results as the horizontal position precision for $4 \mathrm{~m}$, vertical position fro $6 \mathrm{~m}$ and the total position precision for $6.8 \mathrm{~m}$, and timing precision for $16 \mathrm{~ns}$.

\section{CONCLUSION}

A PRSNS with GEO, IGSO and MEO is proposed, and it is simulated by HLA, which can both simulate most of the operating states of the real PRSNS system and measure the technical parameters of the satellite navigation system. The theoretically estimated positioning accuracy of the PRSNS and the results of the data analysis of the experiment were presented. The experimental results were consistent with the estimated positioning accuracy under real conditions, which accord with that of GPS. The presented PRSNS simulation system can greatly benefit the development of the PRSNS.

\section{REFERENCES}

[1] B.Hofmann-Wellenho, Herbert Lichtenegger. GPS Theory and Practice. Fourth revised edition, Springer Wien New York, 1997

[2] Kyle O'Keefe. Evaluation of GALILEO, GPS and Combined System Performance through Simulations. University of Calgary, October, 2001

[3] Francesco Vatalaro, Giovanni Emanuele Corazza, Carlo Caini, Analysis of LEO, MEO, and GEO Global Mobile Satellite Systems in the Presence of Interference and Fading, IEEE Journal on selected areas in communications, vol. 13, No. 2, February 1995, pp. 291-300

[4] Galileo Global Component Architecture, GALAALS-DD-031, Version 3.0, 08.12.00

[5] Zhu Jun, Wen Yuanlan, Liao Ying. Research on the broadcast ephemeris adapted to all kinds of orbits. Aerospace Control, 2005, 23(6):9-11

[6] Kothris D., Beach M., Allen B. and Karlsson P., Performance Assessment of Terrestrial and Satellite Based Position Location Systems[J], 3G Mobile Communication Technologies, 2001, 447(4):26 28

[7] DeRidder, Jean-Jacques and Legenne, Performance of satellite navigation systems in urban areas with augmentations[C], 21st AIAA International Communications Satellite Systems Conference (ICSSC) and Exhibit, Japan, 2003, 149 160 